\title{
Simulando Indenização em Ações Civis por meio de Ontologias Baseadas na Lógica Descritiva
}

\author{
Jean E. S. Araujo ${ }^{1}$, Cleyton M. de O. Rodrigues ${ }^{2}$, Frederico L. G. de Freitas ${ }^{1}$ \\ ${ }^{1}$ Cento de Informática - Universidade Federal de Pernambuco (UFPE) \\ Av. Jornalista Anibal Fernandes, Cidade Universitária - Recife - PE - Brazil \\ ${ }^{2}$ Departamento de Computação - Universidade de Pernambuco (UPE) \\ R. Cap. Pedro Rodrigues, São José - Garanhuns - PE - Brazil \\ jeas@cin.ufpe.br, cleyton.rodrigues@upe.br, fred@cin.ufpe.br
}

\begin{abstract}
Brazilian legal practice has converged to the processing of disputes by electronic means, leading to a progressive decrease in the lawsuits physically processed. The Federal Constitution, in Article 5, item V, ensures indemnity for civil damages arising from the violation of Rights, such as the Right to Life, Freedom, Equality, Security and Property. Therefore, this study presents the OntoDano ontology, which allows (using the Semantic Web standards as Description Logic) to verify whether the case under analysis matches an Indemnifiable Civil Damage. The results achieved so far suggest the possibility of applying the Ontology created in legal/forensic practice.
\end{abstract}

Resumo. A prática jurídica Brasileira tem convergido para o processamento de litígios por meio eletrônico, com a consequente diminuição progressiva das ações processadas fisicamente. A Constituição Federal, no artigo $5^{\circ}$, inciso $V$ assegura a indenização pelo dano cível decorrente da violação de Direitos, como o Direito à vida, à liberdade, à igualdade, à segurança e à propriedade. Portanto, este estudo apresenta a ontologia OntoDano, a qual permite (utilizando os padrões da Web Semântica como a Lógica de Descrições) verificar se o caso em análise corresponde a um Dano Cível Indenizável. Os resultados alcançados até então apontam para a possibilidade da aplicação da Ontologia na prática jurídica/forense.

\section{Introdução}

Muito se discute, nos últimos anos, sobre a importância de uma justiça célere. Afinal, a ausência de celeridade, sob certo ângulo, é ausência de justiça [MONTENEGRO FILHO 2018]. Nesta ótica, ferramentas tecnológicas vêm sendo inseridas no universo jurídico Brasileiro, em especial, abordando estratégias e algoritmos oriundos da Inteligência Artificial. Esta é, portanto, vista como um recurso a ser utilizada em prol da celeridade processual. Importante mencionar que já existem iniciativas da utilização da Inteligência Artificial no judiciário Brasileiro, tais como: o ELIS no $\mathrm{TJPE}^{1}$ que realiza triagem inicial de processos tributários, o Vitor no $\mathrm{STF}^{2}$ que realiza a classificação de processos, e o SINAPSES $^{3}$ voltado para a extração de informações.

\footnotetext{
${ }^{1}$ Tribunal de Justiça do Estado de Pernambuco

${ }^{2}$ Supremo Tribunal Federal

${ }^{3}$ Tribunal de Justiça do Estado de Rondônia
} 
Diferentemente destas iniciativas, o presente trabalho traz um abordagem ontológica para simulação de indenização em ações cíveis. Em particular, a ontologia é aplicada ao Direito Cível Brasileiro, tendo como modelo de referência a OntoCrime [RODRIGUES 2019], uma Ontologia Central para modelar a Teoria Geral do Crime dentro do Direito Penal.Para demonstrar a abordagem ontológica proposta neste trabalho, destacamos ao longo deste estudo os conceitos jurídicos relativos ao Direito Cível, que fundamentam a aplicação ontológica para simulação de indenização em Ações Cíveis.

Este estudo está organizado da seguinte forma. A Seção 2 apresenta brevemente o arcabouço teórico do Direito e do Dano Cível, além do instrumento da Indenização. A Seção 3 destaca a Web Semântica e seus padrões, que foram aplicados na engenharia da ontologia proposta, a qual é apresentada na Seção 4, onde também se demonstram os principais segmentos de sua estrutura. A Seção 5 realiza uma demonstração da aplicação da ontologia OntoDano em cenários reais. Finalmente, a Seção 6 destaca as considerações finais do trabalho realizado e possíveis caminhos para continuidade da pesquisa.

\section{Direito Cível}

O Direito Cível é um segmento extenso do ordenamento jurídico brasileiro. No entanto, alguns conceitos são fundamentais para a compreensão da abordagem proposta neste trabalho. A identificação da situação de indenização diante de algum evento de relevância para o meio jurídico tem embasamento inicial no ato ilícito, que se origina de fato jurídico compreendendo todo acontecimento capaz de produzir consequências jurídicas [MELLO 2015]. Todavia, não basta a existência de uma legislação que defina o ato ilícito, a obrigação de reparar nasce da culpa e não do dano. Nesse entendimento, afirma-se que o principal pressuposto da responsabilidade é a Culpa [IHERING 1974] (a atribuição do dever de indenizar sem a presença da culpa possui legislação específica). Em continuidade, analisando o código cível do ordenamento jurídico, a responsabilidade cível que antecede o instituto da indenização possui os seguintes pressupostos:

- CULPA: corresponde a uma predisposição mental do agente do dano, o que fica evidente no seguinte dispositivo legal "Aquele que, por ação ou omissão voluntária, negligência ou imperícia, violar direito ou causar prejuízo a outrem, fica obrigado a reparar o dano";

- NEXO CAUSAL: vínculo existente entre a conduta do agente e o resultado por ela produzido;

- DANO: revelado na expressão "violar direito ou causar prejuízo a outrem."

Outro aspecto de grande relevância no Dano Cível e a consequente atribuição da Indenização está no requisito da Vítima ser distinta do Autor do Dano. Caso contrário, seria como admitir que o Autor vai indenizar a si mesmo por um eventual Dano cometido.

Considerando a aplicação de Ontologias no Direito Cível, destacam-se dois trabalhos relacionados. Almeida e Oliveira [ALMEIDA and OLIVEIRA 2014] propõem a utilização de uma ontologia para mapear relacionamentos do Direito Cível em prol de uma visão clara, concisa e inequívoca do domínio, promovendo a interoperabilidade com outros sistemas. Laarschot et al. [LARRRSCHOT et al. 2005] descrevem uma ontologia para amenizar discrepâncias de termos usados entre profissionais do Direito e documentos jurídicos, suportando ainda inferências de normas que determinem responsabilidades civis das partes envolvidas nos casos. 


\section{Segmento da Inteligência Artificial}

Nesta seção apresentamos uma breve explanação do fundamento teórico de Inteligência Artificial que estrutura o processo de simulações de indenizações por Dano em Ações Cíveis. E para tanto, discorremos sobre Lógica de Descrições, Web Semântica e Ontologias.

\subsection{Lógica de Descrições}

A Lógica de Descrições (ou Lógica Descritiva, do inglês, Description Logic, DL) é um formalismo para representação do conhecimento com suporte à tarefas de raciocínio. É segmentada em categorias que se diferenciam pelo poder de expressividade, o qual se relaciona diretamente com a complexidade das inferências realizadas. Em consequência, observa-se a relação de quanto maior a complexidade, maior o tempo para retorno do processo de inferência [F. BAADER 2003].

A expressividade da linguagem da Lógica de Descrições está atrelada ao conjunto de construtores e propriedades disponíveis para representar algum domínio. Há, entretanto, um conjunto de construtores comuns a todos os tipos da Lógica de Descrições. Assim, considerando que $A$ e $B$ sejam conceitos e $P$ seja um papel (propriedade), destacam-se os seguintes construtores básicos: Conjunção $(A \sqcap B)$; Disjunção $(A \sqcup$ $B)$; Quantificação Universal $(\forall P . A)$; Quantificação Existencial ( $\exists$ P.A); e, Negação: $(\neg A)$. Importante destacar a possibilidade da separação da base de conhecimento nos componentes TBox e ABox, sendo que o TBox estabelece a estrutura de conceitos, ou seja, o conhecimento intencional (onde encontram-se a inclusão $A \sqsubseteq B$, e equivalência de conceitos $A \equiv B$ ) e o $A B o x$ são as instâncias relativas ao vocabulário (conhecimento extensional). Considere, por exemplo, a definição do conceito Pai e do papel temFilho. Tem-se, a seguir, uma exemplificação do que seria uma amostra do ABox:

- Conceito: Pai(joao) - declara que João é uma instância do conceito Pai;

- Papel: temFilho(joao, matheus) - declara uma instância em que João tem um filho Matheus.

Neste presente trabalho, a expressividade da linguagem $\mathcal{A L C I} \mathcal{Q}(\mathcal{D})$ atende ao escopo da representatividade e inferências requeridas pela OntoDano, a qual, além dos construtores mencionados, possibilita: propriedades inversas $\left(\mathcal{P}^{-}\right)$, restrições de cardinalidade qualificadas $\mathcal{Q}$, e dados tipados em valores e propriedades $\mathcal{D}$ [F. BAADER 2003].

Já a semântica da Lógica de Descrições é definida em termos de interpretações de primeira ordem $\mathcal{I}$, ou seja, uma tupla $\left(\Delta^{\mathcal{I}},{ }^{\mathcal{I}}\right)$ onde o primeiro elemento corresponde ao domínio de $\mathcal{I}$ e ${ }^{\mathcal{I}}$ é uma função de interpretação, que faz o mapeamento de conceitos para subconjuntos de $\Delta^{\mathcal{I}}$, bem como, o mapeamento de papéis para subconjuntos de $\Delta^{\mathcal{I}} \mathrm{x} \Delta^{\mathcal{I}}$, e instâncias para elementos de $\Delta^{\mathcal{I}}$.

\subsection{Web Semântica e Ontologias}

A Web Semântica é fruto da evolução da Web Original (ou web sintática, ou web 1.0), na qual os conteúdos ou recursos disponibilizados na Web não estão mais isolados. Isto é, dispondo de uma camada semântica para estes recursos, eles podem relacionar-se com outros conteúdos gerando novos (e até então, implícitos) componentes de conhecimentos. Isto ocorre porque a semântica permite que computadores e homens possam compreender 
o significado destes recursos, amenizando potenciais anomalias comuns de linguagens naturais, como conceitos polisêmicos e ambiguidades referenciais. Esta nova realidade da web possibilita soluções de busca, indexação e recuperação de informações mais complexas [BERNES-LEE 2000], bem como tarefas de raciocínio para criação de serviços mais sofisticados.

$\mathrm{Na}$ prática, a Web Semântica se fundamenta em modelos conceituais conhecidos como ontologias [BERNES-LEE 2003]. Na computação, uma Ontologia é uma especificação explícita e formal de uma conceitualização compartilhada [STUDER et al. 2004]. A OWL (do inglês, Ontology Web Language) é a linguagem recomendada para representar o conhecimento rico e complexo sobre coisas, grupo de coisas e relações entre coisas [GRAU et al. 2008]. O tema Ontologia abrange uma diversidade de aplicações, metodologias de construção, tipos e finalidades [ALMEIDA and BAX 2003].

Os componentes mais relevantes em uma ontologia são: (i) Conceito, ou seja, termos, itens do vocabulários de algum domínio específico (Parte, Crime, Lei); (ii) Papel, que relaciona os conceitos, por exemplo, o papel comete que relaciona os conceitos Parte e Crime; e, (iii) Axiomas, que são regras formalizadas dentro de algum domínio representado, como a regra em que um crime envolve duas partes, a ativa e a passiva.

Como ontologia superior, utilizamos a UFO (Unified Foundational Ontology) [GUIZZARDI 2005] [GUIZZARDI et al. 2013], uma ontologia Formal baseada em teorias abrangentes da Lógica Filosófica. A UFO faz uso de conceitos gerais, comuns a vários domínios, permitindo a interoperabilidade semântica entre subdomínios. Portanto, esse processo de alinhamento da OntoDano à uma ontologia Superior facilita a ampliação do modelo para outros conceitos.

\section{OntoDano}

A OntoDano é a ontologia de domínio acerca do Dano Cível, e tem seu referencial conceitual baseado na OntoCrime [RODRIGUES 2019], uma Ontologia Central para modelar a Teoria Geral do Crime. Desta forma, aproveitamos a estrutura da ontologia similar, ajustando para representar o domínio de conhecimento referente ao Dano Cível, correspondente à indenização. Por a OntoCrime usar a UFO como ontologia formal de Topo, a OntoDano indiretamente também estende a estrutura formal da UFO, bem como está de acordo com o tipologia de conceitos universais adotada.

O processo do levantamento de conhecimento acerca do Dano Cível e a correspondente indenização foi realizado seguindo duas diretivas: Pesquisa de sentenças e Pesquisa Quantitativa através de um formulário disponibilizado para especialistas da área. Foi selecionado, portanto, um conjunto de sentenças dentro do área de atuação das pessoas que responderam a pesquisa de termos e conceitos relacionados ao Dano Cível. Em relação às sentenças, foram descartadas da análise do conteúdo, aquelas que não tinham procedência total com o petitório ajuizado, bem como, as que correspondiam a homologação de acordos. Tal descarte se deve ao fato que na procedência parcial apenas parte do pedido ajuizado foi concedido e esse juízo de valor se utiliza de critério de subjetividade que torna a análise mais complexa dentro da perspectiva da lógica. O que ocorre também com a homologação de acordo, já que nessa homologação pode haver uma dispensa de direitos por uma das partes do processo, também, dentro de um critério de subjetividade para valoração da viabilidade por qualquer da partes envolvidas no processo. 
Para efeitos de simplificação, a estrutura semântica da ontologia será demonstrada através de um diagrama de classes na notação Unifed Modelling Language (UML), onde conceitos são tratados como classes, relações de subsunções são heranças entre as classes, e as relações binárias apresentam-se como associações. A apresentação da ontologia OntoDano será apresentada em módulos, destacando os elementos advindos da OntoCrime (OC) que, por sua vez, se encaixam na estrutura formal da UFO.

\subsection{Objetos do Dano}

A Figura 1 ilustra os objetos da OntoDano que mantém a estrutura lógica já definida na OntoCrime. Ressaltando que, na esfera cível do Dano, existe uma dicotomia que merece ser destacada, a qual seja, dos Danos Patrimoniais e os Extrapatrimoniais, uma vez que o Dano Extrapatrimonial está atrelado a um Objeto Moral.

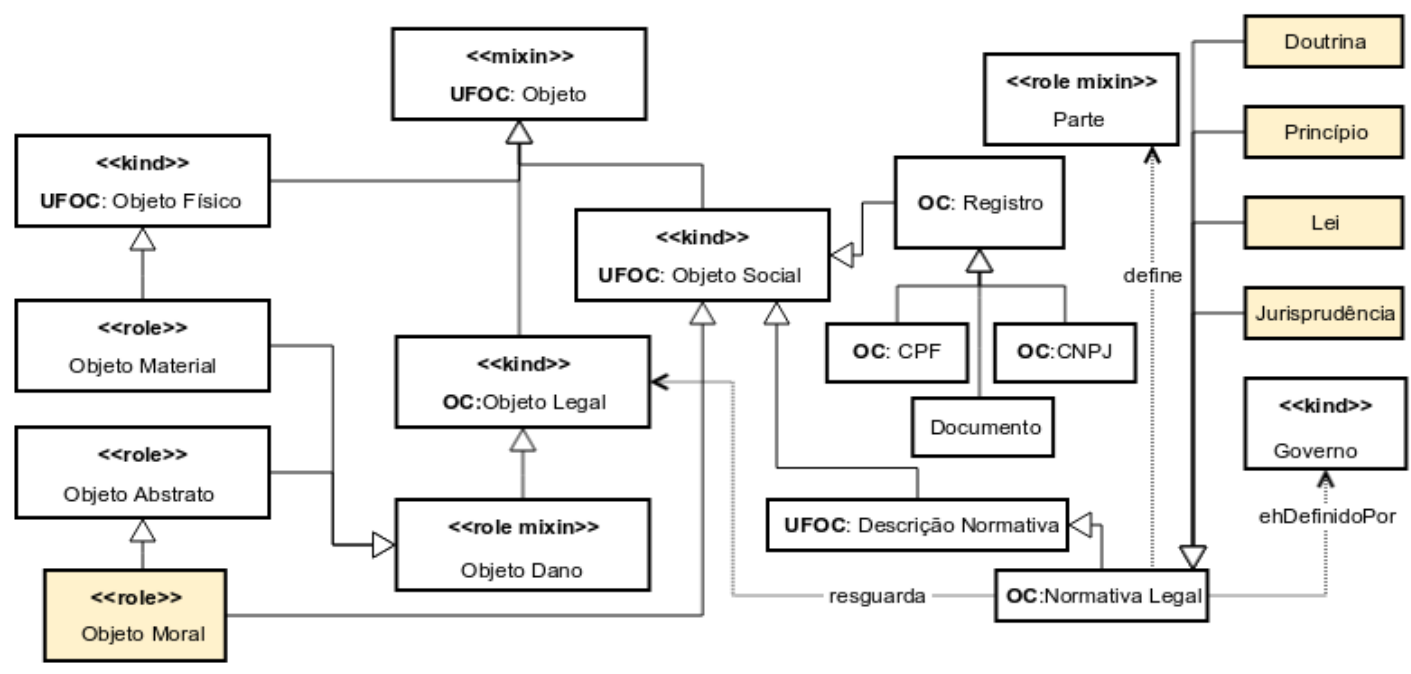

Figura 1. Objetos da OntoDano. Fonte: Adaptado de Rodrigues (2019)

A Normativa Legal representa o ordenamento jurídico brasileiro que trata do Direito Cível. Também, o Dano e a orientação sobre a respectiva da Indenização não é respaldada apenas nas Leis do Direito Positivo, mas é lastreada por embasamento formados por jurisprudências (conjunto das soluções dadas pelos tribunais sobre as questões de direito similares ao caso analisado), doutrinas (ideias e ensinamentos de autores e juristas que fundamentam as decisões judiciais), princípios (enunciados normativos com teor universal que orientam a compreensão do ordenamento jurídico), além das próprias leis.

\subsection{Agentes do Dano}

A Figura 2 demonstra a estruturação dos conceitos relacionados aos agentes na ontologia OntoDano, ou seja, aqueles envolvidos num fato jurídico correspondente ao Dano na esfera do Direito Cível e sua Indenização. As definições e relacionamentos de agentes na OntoCrime foram reusadas com adaptações, destacando que a divergência no quesito de agente refere-se ao fato que no Direito Cível o AgenteInstitucional pode ser responsabilizado por um Dano cometido por um AgenteAtivo sob sua responsabilidade. Desta forma, o ResponsávelDano pode ser endereçado a uma PessoaFísica que seja CivilmenteCapaz. e que possua algum AgenteInstitucional que responda por este. 


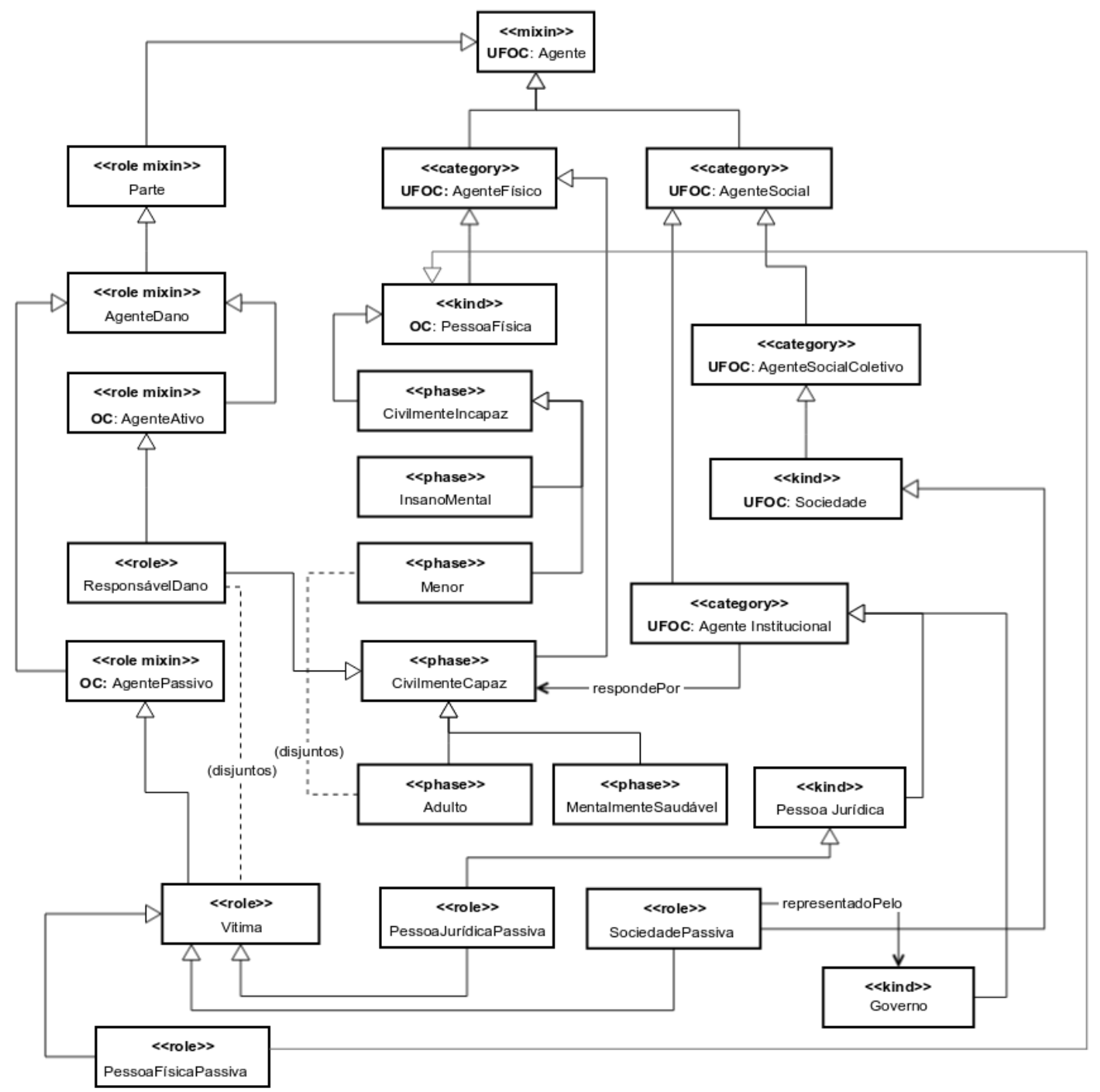

Figura 2. Agentes da OntoDano. Fonte: Adaptado de Rodrigues (2019)

O Dano possui a polaridade entre a posição ativa versus a passiva na prática da lesão ao bem, e sobre esse esteio se acopla os conceitos da UFO-C acerca da entidade Agente. Destacamos, a seguir, as principais informações dos elementos que compõem os conceitos subsumidos:

- Parte corresponde ao LegalAgent da OntoCrime. A opção por essa nomenclatura se deve ao termo ser mais usual no segmento do Direito Cível;

- AgenteDano corresponde ao CrimeAgent da OntoCrime. Esse conceito se polariza em AgenteAtivo e AgentePassivo, os quais, respectivamente se enquadram no ResponsávelDano e na Vítima;

- PessoaFísica corresponde ao PessoaNatural da OntoCrime. Esse conceito se ramifica em CivilmenteCapaz e CivilmenteIncapaz. Nesse ponto, guarda uma distinção para a OntoCrime. Essa divisão se mostrou adequada para a OntoDano para vincular o CivilmenteCapaz que comete um Dano investido de função pública 
ou de empregado em que a responsabilidade de responder, ou melhor, indenizar recai sob o Agente Institucional correspondente, Governo ou Pessoa Jurídica;

- ResponsávelDano tem uma correspondência relativa com o CrimeAutor. Na esfera Penal, não há possibilidade da pena recair sobre um Agente Institucional, o que pode haver são repercussões de um evento Penal na esfera Cível. Bem como há uma disjunção entre o ResponsávelDano e a Vítima que correspondem aos polos antagônicos AgenteAtivo e AgentePassivo. Uma situação especial que difere na esfera Penal, por exemplo, o evento do suicídio é um caso particular em que o AgenteAtivo e o AgentePassivo se endereçam na mesma PessoaFísica.

- Vítima é um conceito disjunto de ResponsávelDano, ressaltando que um evento danoso quando tem a contribuição da vítima exclui a possibilidade de indenização.

Os conceitos CivilmenteCapaz e CivilmenteIncapaz e os subsumidos por estes são enquadrados como phases (fases) que são elementos ontológicos do tipo anti-rígidos, já que dependem unicamente de propriedades intrínsecas da PessoaFísica a qual instanciam e podem mudar no período de existência da PessoaFísica. Outro conceito de relevância na caracterização do Dano Cível Indenizável é o Nexo Causal que será detalhado a seguir.

\subsection{O Nexo Causal}

De acordo com Lehmann et al. [LEHMANN et al. 2004], a Causalidade é a ocorrência de dois eventos, a causa e o efeito, destacando que o objeto que participa do cenário de Causalidade não é o mesmo entre a Causa e o Efeito. A Figura 3 demonstra os relacionamentos envolvidos na conceituação do Nexo Causal.

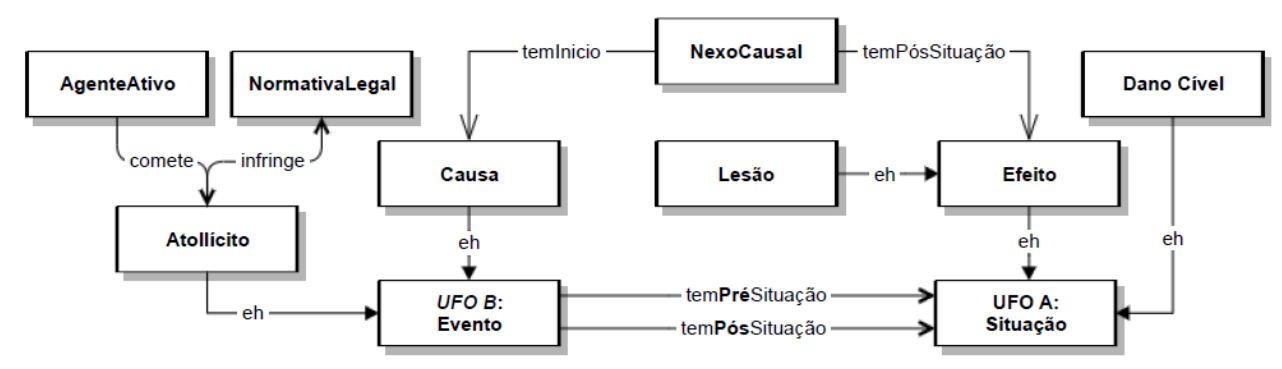

Figura 3. Nexo Causal. Fonte: própria.

O Dano Cível Indenizável é uma Situação que tem como pressuposto para sua caracterização o Nexo Causal. Na inexistência do Objeto eventualmente lesado, não há o que se falar em Nexo Causal e, por consequência, em Indenização. Objeto, como se observa na Figura 1, é um conceito subsumido de Substantial (UFO). A Figura 3 expõe a existência de duas situações distintas: a anterior ao evento danoso, e a posterior que se configura como o Efeito. O evento que ocorre entre essas duas situação é justamente o que se enquadra como a Causa. O vínculo entre a Causa e o Efeito é denominado de Nexo Causal. Outras informações importantes da modelagem da OntoDano são elencadas em combinação ao que pode ser observado na Figura 3 em cenário jurídico que se estabelece a Indenização pelo Dano Cível:

- A Vítima não contribui para a ocorrência do Dano;

- A Vítima e o Agente não podem ter a mesma instância, são conceitos disjuntos;

- Após a ocorrência do Evento danoso, a Situação antecede ao Evento tem que ser distinta da Situação que sucede. Se nada muda, não há Dano nem há o que se falar em Nexo Causal. 


\subsection{Axiomatizações da OntoDano}

Nesta seção, destacamos a representação dos principais conceitos e relações da OntoDano

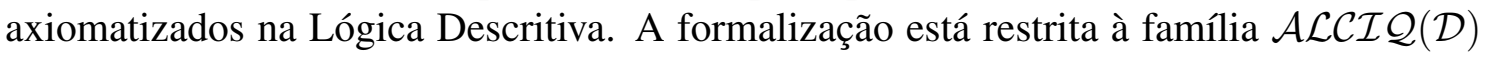
pois já atende ao esforço deste trabalho para registrar cenários jurídicos que apontem para o instituto da Indenização em Danos da esfera do Direito Cível. Ressalta-se, entretanto, que não está esgotada toda a representação ontológica da OntoDano em questão. Os axiomas relacionados aos agentes e objetos estão representados em $\mathcal{T}_{a g-o b}$.

$$
\mathcal{T}_{\text {ag-ob }}:\left\{\begin{array}{l}
\text { ヨtemRegistro.CPF } \\
\text { PessoaJuridica } \\
\text { CivelmenteCapaz } \\
\text { CivelmenteIncapaz } \\
\text { CivelmenteIncapaz } \\
\text { Adulto } \\
\text { MentalmenteSaudavel } \\
\text { AgenteAtivo } \\
\text { AgentePassivo } \\
\text { AgenteAtivo } \\
\text { AgenteAtivo } \\
\text { ResponsavelDano } \\
\text { Leis } \\
\text { Leis } \\
\text { NormativaLegal } \\
\text { ObjetoDano } \\
\text { ObjetoMaterial } \\
\text { ObjetoAbstrado } \\
\text { ObjetoDano } \\
\text { ObjetoLegal } \\
\text { AgenteLegal } \\
\text { define } \\
\text { comete } \\
\text { temResponsavel } \\
\text { respondePor }
\end{array}\right.
$$

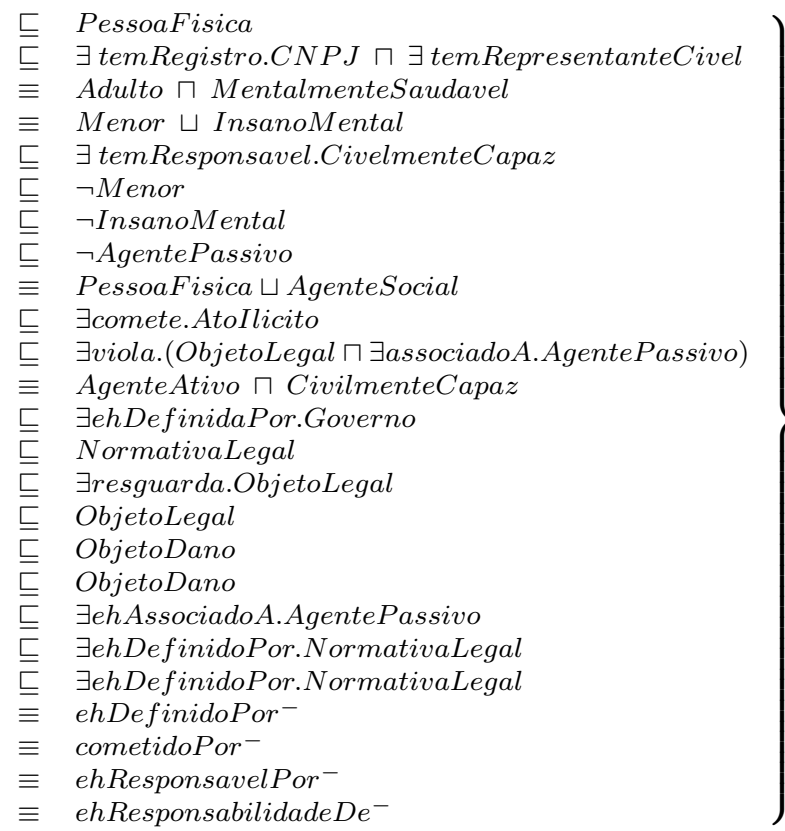

A formalização para o Dano Cível e os pressupostos para a caracterização de um Dano Indenizável está destacada em $\mathcal{T}_{\text {dano }}$.

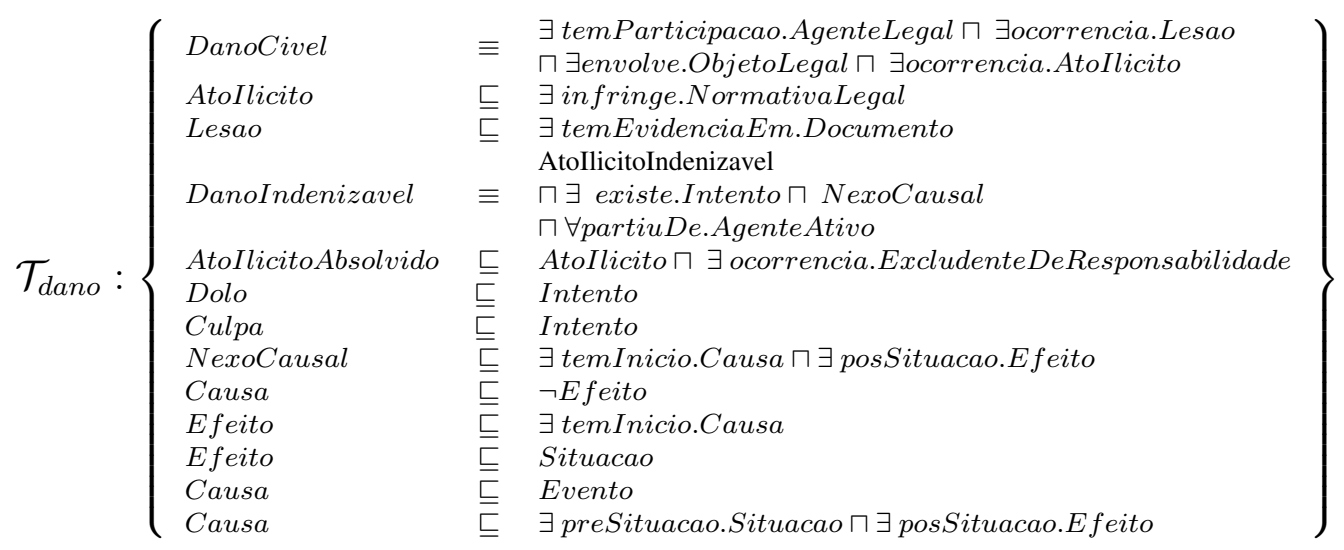

\section{Estudo de Casos}

Esta seção se propõe a apresentar casos de uso que demonstram a aplicação da OntoDano em cenários jurídicos reais resultantes da pesquisa de sentenças. Optamos por uma notação mais intuitiva para as ilustrações dos casos explanatórios que visam representar visualmente o cenário jurídico de Dano Cível Indenizável que obteve no processo de raciocínio a mesma sinalização, ou seja, o cenário representado corresponde a um Dano Cível Indenizável. 


\subsection{Cobrança Vexatória}

A Figura 4 esboça o cenário selecionado que trata de caso de Indenização por Dano Moral.

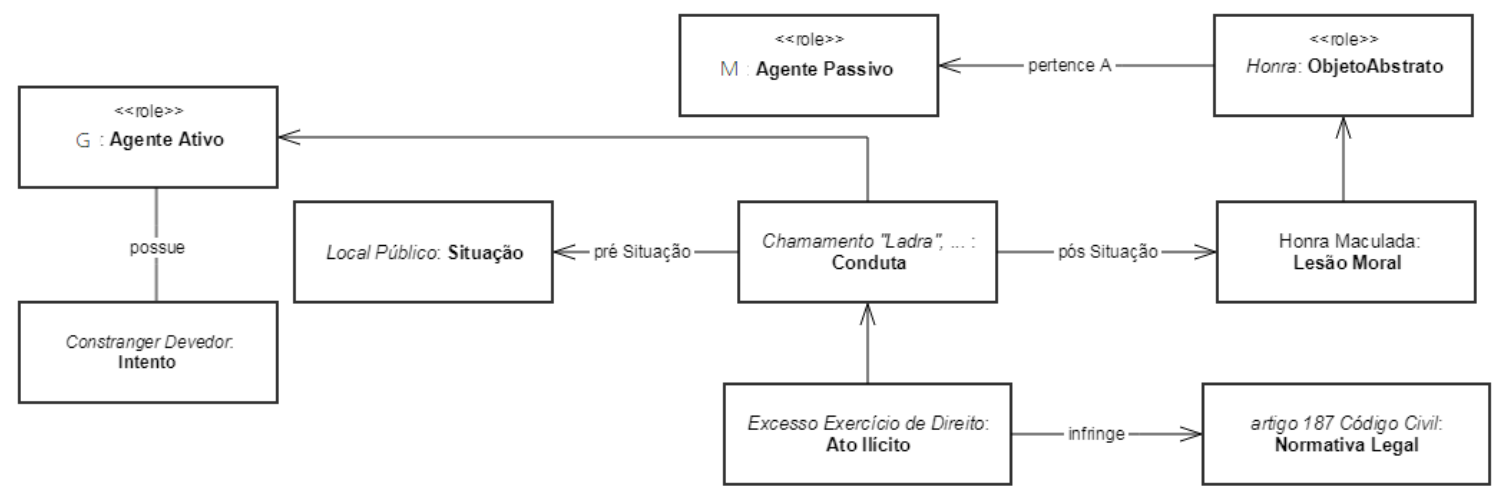

Figura 4. Caso 1: Cobrança Vexatória. Fonte: própria.

O caso em tela corresponde a uma cobrança vexatória que corresponde a situação em que o credor cobra o devedor de maneira desonrosa, excedendo aos limites ao direito patrimonial em seu fim econômico ou social como preconiza o artigo 187, CC/2002. Utilizando os dados de processo ${ }^{4}$, segue a instanciação da OntoDano e resultado das inferências realizadas. Estas foram aplicadas com as instâncias ABox representadas na Figura 4, resultando na indicação do Dano Cível Indenizável ${ }^{[8]}$ com a indicação de Genésio $(G)$ para o Responsável do Dano ${ }^{[2]}$. Serão demonstrados alguns passos de inferência em DL sobre o caso em análise de Cobrança Vexatória. Para melhor visualização, alguns termos foram abreviados. ${ }^{5}$

$$
\begin{aligned}
& {[1] \frac{\left\{\begin{array}{l}
\text { Adulto(genesio }), \text { MentalmenteSaudavel }(\text { genesio }), \\
\text { CivilmenteCapaz } \equiv \text { Adulto } \sqcap \text { MentalmenteSaudavel }
\end{array}\right\}}{\models \text { CivilmenteCapaz }(\text { genesio })}} \\
& 2] \frac{\left\{\begin{array}{l}
\text { comete }(\text { genesio, } \text { (mlcp }), \text { AtoIlicito }(\text { cmlcp }), \text { CivilmenteCapaz }(\text { genesio })^{[1]}, \\
\text { ResponsavelDano } \equiv \exists . \text { comete.AtoIlicito } \sqcap \text { CivilmenteCapaz }
\end{array}\right\}}{\models \text { ResponsavelDano }(\text { genesio })}
\end{aligned}
$$

O passo 2 demonstra que Genesio é o responsável pelo Dano.

$$
\begin{gathered}
{[3] \frac{\{\text { Lesao }(\text { hm }), \text { Lesao } \sqsubseteq \text { Efeito }\}}{\models \text { Efeito }(\text { hm })}} \\
{[4] \frac{\{\text { AtoIlicito }(\text { cmlcp }), \text { AtoIlicito } \sqsubseteq \text { Evento }\}}{\models \text { Evento }(\text { cmlcp })}}
\end{gathered}
$$
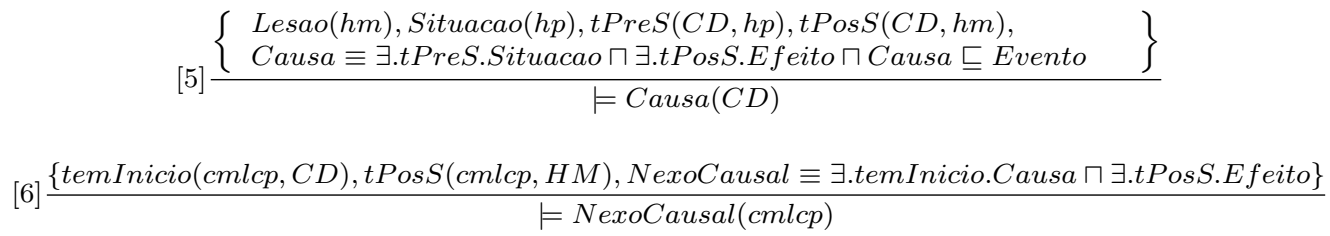

\footnotetext{
${ }^{4}$ 5178-48.2013.8.17.0640/TJPE

${ }^{5}$ cmlcp: Chamar Marlene de Ladra e Caloteira em Publico; hm: Honra Maculada; hp: Honra Preservada; tPreS: tem Pré Situação; tPosS: tem Pós Situação; CD: Cobrar Devedor; gpc: Gravação por Câmeras
} 


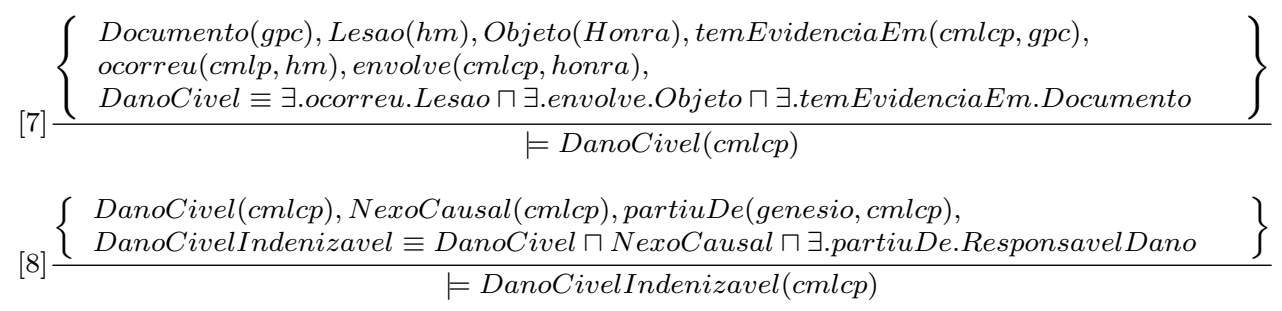

No passo 8 fica demonstrado a inferência que a situação de Chamar Marlene de Ladra e Caloteira em Público corresponde a um Dano Cível Indenizável.

\subsection{Negativação Indevida}

A Figura 5 esboça um novo cenário que trata de caso da Indenização por Dano Moral IN RE IPSA, em virtude de Negativação Indevida.

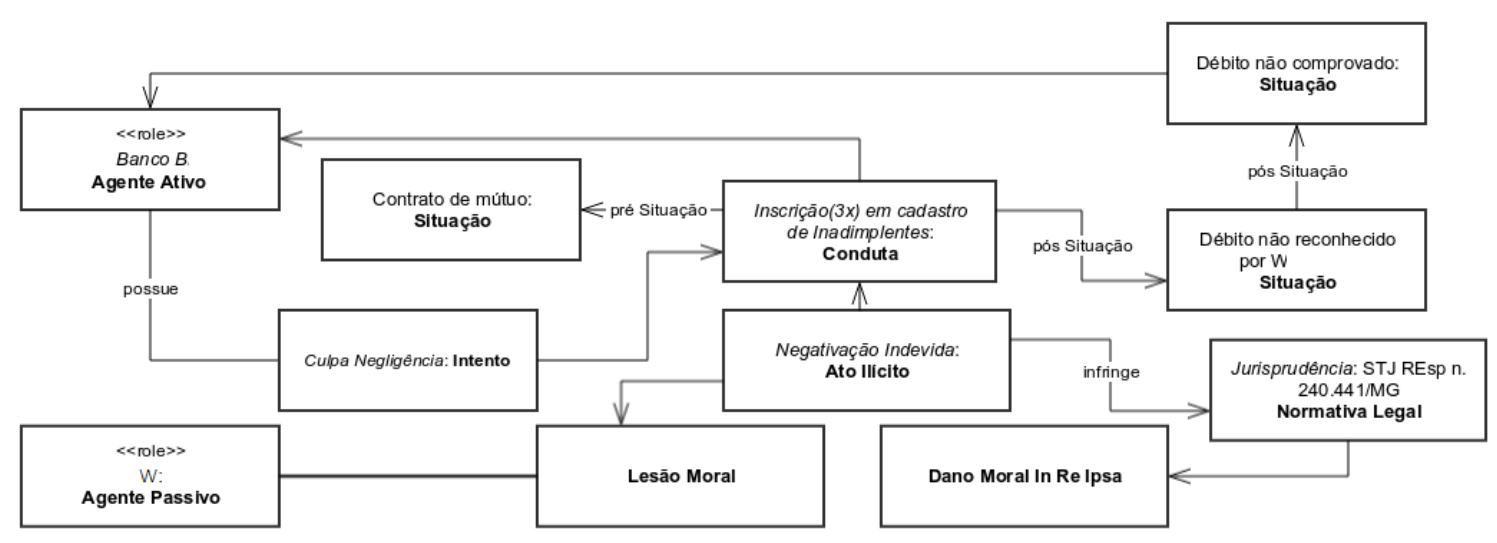

Figura 5. Caso 2: Negativação Indevida. Fonte: própria.

O caso corresponde a outro cenário ${ }^{6}$ jurídico analisado. Trata-se da imputação de débito não comprovado com Negativação Indevida que configura um Dano Moral In Re Ipsa, tido como presumido, ou seja, independe da comprovação do grande abalo psicológico sofrido pela vítima. A inferência aplicada ao cenário também demonstra a indicação do Dano Cível Indenizável e como Responsável do Dano, o banco Bradesco. Igualmente, segue amostra de passos de inferência em DL sobre instâncias do caso em análise. ${ }^{7}$

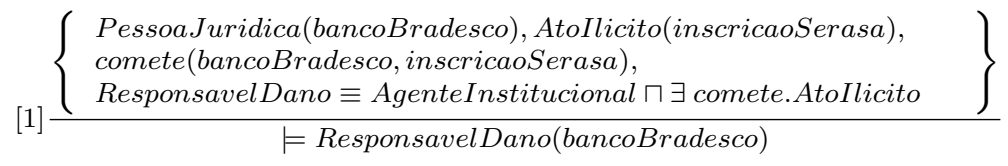

Esse passo de inferência demonstra como responsável do Dano, o bancoBradesco. A identificação do responsável do dano é um pressuposto para a Indenização do Dano Cível.

\footnotetext{
$\{\operatorname{situacao}(n i)$, LesaoMoral $($ lmi $)$, Documento $($ dnc $)$, Objeto(moral $)$,

DanoCivel $\equiv \exists$ ocorreu.Lesao $\sqcap \exists$ temEvidenciaEm.Documento $\sqcap \exists$ envolve.ObjetoLegal, ocorreu(ni,lmi), temEvidenciaEm(ni,dnc), envolve(ni, moral)

$[2] \stackrel{\operatorname{DanoCivel}(\mathrm{ni})}{ }$

64877-04.2013.8.17.0640/TJPE

${ }^{7}$ Abreviações ni: Negativação Indevida; dnc: Débitos Não Comprovados; lmi: Lesão Moral IN RE IPSA
} 


$$
\begin{aligned}
& {[3] \frac{\{\text { AtoIlicito }(\text { inscricaoSerasa }), \text { AtoIlicito } \sqsubseteq \text { Evento }\}}{\models \text { Evento }(\text { inscricaoSerasa })}}
\end{aligned}
$$

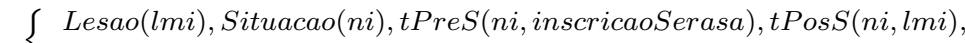

$$
\begin{aligned}
& \left.[4] \frac{\{\text { Causa } \equiv \exists . t \text { PreS.Situacao } \sqcap \text { ヨ.tPosS.Efeito } \sqcap \text { Causa } \sqsubseteq \text { Evento }}{\models \text { Causa(inscricaoSerasa })}\right\}
\end{aligned}
$$

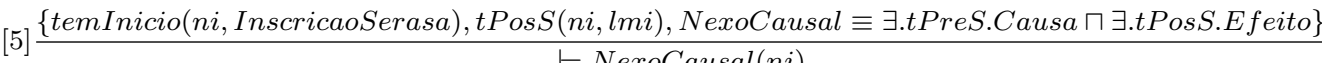

$$
\begin{aligned}
& =\text { NexoCausal }(\text { ni })
\end{aligned}
$$

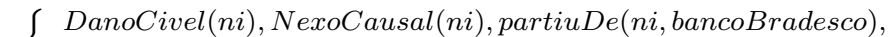

$$
\begin{aligned}
& {[6] \frac{\{\text { DanoCivelIndenizavel } \equiv \text { DanoCivel } \sqcap \text { NexoCausal } \sqcap \exists . p a}{\models \text { DanoCivelIndenizavel }(\text { ni })}}
\end{aligned}
$$

Com o passo 6 tem a finalização da demonstração de que a situação de negativação indevida é um Dano Cível Indenizável.

\section{Considerações}

A formalização do conhecimento jurídico por meio da representação ontológica não é uma tarefa simples, em virtude da diversidade de textos jurídicos que tratam do mesmo assunto. Ainda assim, existem núcleos de conhecimento que estão pacificados. De maneira geral, o segmento do Direito Cível que define o núcleo do Dano Cível está consolidado. A esse núcleo que a OntoDano faz referência, registrando as características que fazem parte da regra geral para definição do Dano Cível e correspondente Indenização na abordagem ontológica que aqui fica denominada de OntoDano.

Diante disso, a aplicação prática da OntoDano em casos práticos requer a criação de ontologias derivadas a exemplo das Figuras 4 e 5. O tema Direito Cível é um ramo jurídico amplo e aparece entre os cinco assuntos com os maiores quantitativos de processos em todas as instâncias da Justiça Estadual, como se pode observar no relatório de Justiça em Números do ano de 2019 [CNJ 2019]. Em especial, a indenização por Dano Moral corresponde a mais de $5 \%$ de todas as ações ajuizados no sistema jurídico Brasileiro.

Como trabalhos futuros, almeja-se realizar investigações empíricas da OntoDano, com especialistas da área do Direito, para entender o grau de aproximação da ontologia com a realidade. Em particular, é estimado que a aplicação desse trabalho tenha maior relevância em juizados especiais, em virtude, da menor complexidade das demandas ajuizadas e do próprio rito que possui como um dos atributos, a celeridade. Desta forma, faz-se necessário, derivações da OntoDano em cenários específicos do Direito Cível, aqui sugeridos, no ambiente dos Juizados Especiais. No entanto, esse trabalho pode ser estendido com a utilização de Lógicas Descritivas Preferencias [FERREIRA 2012] para alcançar complexidades jurídicas no âmbito do Direito Cível que não foram estudadas, a exemplo, da procedência parcial do litígio.

\section{Referências}

ALMEIDA, M. and BAX, P. (2003). Uma visão geral sobre ontologias: pesquisa sobre definições, tipos, aplicações, métodos de avaliação e de construção. Ci. Inf., Brasília, 2003.

ALMEIDA, M. and OLIVEIRA, J. (2014). A legal ontology of relationships for civil law system. CEUR-WS, 2014. 
BERNES-LEE, T. (2000). Semantic Web on XML. Keynote presentation for XML.

BERNES-LEE, T. (2003). Ontologias e a Web Semântica. Anais do XXIII. Congresso da Sociedade Brasileira de Computação - Campinas.

CNJ (2019). Relatório da justiça em números 2019. https://www.cnj.jus.br/pesquisasjudiciarias/justica-em-numeros/.

F. BAADER, D. CALVANESE, D. M. M. D. N. (2003). The Description Logic Handbook: Theory, Implementation and Applications. Cambridge. Cambridge.

FERREIRA, F. (2012). Expressividade e Complexidade em Lógicas Preferenciais, Híbridas e de Grau Limitado. PhD thesis, Fortaleza.

GRAU, B., HORROCKS, I., MOTIK, B., BIJAN, B., P. SCHNEIDER, P., and SATTLER, U. (2008). Owl 2: The next step for owl. volume 6, pages 309-322. Elsevier Science Publishers B. V., Amsterdam, The Netherlands.

GUIZZARDI, G. (2005). Ontological foundations for structural conceptual models. PhD thesis, Enschede.

GUIZZARDI, R., BARCELOS, P., GARCIA, A., and BAX, P. (2013). An ontology reference model for normative acts. 7th Brazilian seminar on ontological research (ONTOBRAS), 2013.

IHERING, M. (1974). Estudios Juridicos. Posada. Estudios Juridicos.

LARRRSCHOT, R. V., Steenbergen, W. V., Stuckenschmidt, H., Lodder, A. R., and van Harmelen, F. (2005). The legal concepts and the layman's terms - bridging the gap through ontology-based reasoning about liability. In JURIX, volume 134 of Frontiers in Artificial Intelligence and Applications, pages 115-125. IOS Press.

LEHMANN, J., BREUKER, J., and BROUWER, B. (2004). Intelligence and Law. Springer Berlin Heidelberg - Rome.

MELLO, M. (2015). Teoria do Fato Jurídico. Plano das Validade. Editora Saraiva, 14th edition.

MONTENEGRO FILHO, M. (2018). Novo Código de Processo Civil Comentado. Editora Atlas LTDA, 3th edition.

RODRIGUES, C. (2019). Uma Abordagem Ontológica para Simulação de Ação Legal e Consistência Semântica Aplicada à Legislação Brasileira. Recife. Centro de Informática, UFPE.

STUDER, R., SURE, Y., and STAAB, S. (2004). Handbook on Ontologies. Springer Berlin Heidelberg - Berlin. 